\title{
Laser-triggered ion acceleration and table top isotope production
}

\author{
K. Nemoto \\ Central Research Institute of Electric Power Industry, 2-11-1, Iwado-kita, Komae-shi, \\ Tokyo 201-8511, Japan
}

A. Maksimchuk, ${ }^{\text {a) }}$ S. Banerjee, K. Flippo, G. Mourou, and D. Umstadter

Center for Ultrafast Optical Science, University of Michigan, Ann Arbor, Michigan 48109-2099

V. Yu. Bychenkov

P. N. Lebedev Physics Institute, Russian Academy of Science, Moscow 117924, Russia

(Received 5 June 2000; accepted for publication 22 November 2000)

\begin{abstract}
We have observed deuterons accelerated to energies of about $2 \mathrm{MeV}$ in the interaction of relativistically intense $10 \mathrm{TW}, 400$ fs laser pulse with a thin layer of deuterated polystyrene deposited on Mylar film. These high-energy deuterons were directed to the boron sample, where they produced $\sim 10^{5}$ atoms of positron active isotope ${ }^{11} \mathrm{C}$ from the reaction ${ }^{10} \mathrm{~B}(d, n){ }^{11} \mathrm{C}$. The activation results suggest that deuterons were accelerated from the front surface of the target.

(c) 2001 American Institute of Physics. [DOI: 10.1063/1.1343845]
\end{abstract}

Since their invention more than sixty years ago cyclotrons ${ }^{1}$ have been the standard method used to accelerate protons and ions for experiments in nuclear physics and for applications in nuclear medicine. The development of shortpulse high-intensity lasers ${ }^{2}$ made it possible to accelerate protons and heavier ions to multi- $\mathrm{MeV}$ energies in the interaction of these lasers with solid targets, ${ }^{3-6}$ gas jets, ${ }^{7}$ and clusters. ${ }^{8}$ It was shown that these accelerated protons can induce nuclear transformations. ${ }^{6}$ In this letter we report on isotope production with deuterons accelerated to millionelectron-volt energies by a powerful laser beam. The results of this study shed new light on the mechanism of ion acceleration and imply that light-ion beams can also be used for advanced inertial confinement fusion research or as a highcurrent injector for linear accelerators.

In previous studies ${ }^{5}$ with the same laser system, a 1.5 $\mathrm{MeV}$ proton beam was observed in the forward direction from aluminum targets using a high-intensity high-contrast $0.53 \mu \mathrm{m}$ illumination. The protons were found to originate from a water contamination layer on the surface of $\mathrm{Al}$ foils. Proton acceleration was attributed to the electrostatic field of charge separation due to "vacuum heating," 9,10 which occurs when electrons are accelerated by the laser electric field at the sharp vacuum-target interface and deposit their energy inside the target. Similar experiments conducted at higher laser intensities at $1 \mu \mathrm{m}$ have been reported elsewhere. ${ }^{4,6} \mathrm{In}$ one case, energy-dependent ring structure of the proton beam was attributed to self-generated magnetic fields and a frontside origin of accelerated protons was claimed. ${ }^{4}$ In the other case, the use of a wedged target allowed the authors to conclude a proton rear-side origin. ${ }^{6}$

In order to better understand the mechanism for deuteron acceleration, we revisited our earlier experiments on proton acceleration, but under different conditions of laser illumination (i.e., $1 \mu \mathrm{m}$ instead of $0.5 \mu \mathrm{m}$ ). In these experiments we have used a Mylar $\left(\mathrm{H}_{8} \mathrm{C}_{10} \mathrm{O}_{4}\right)$ foil which contains more hy-

a)Electronic mail: tolya@umich.edu drogen than the contamination layer on a surface of $\mathrm{Al}$ target and thus is expected to be a more dense proton source. The experiments were performed with a 10 TW hybrid Ti:sapphire/Nd:phosphate glass laser, based on the chirped pulse amplification technique. The system is able to deliver up to $4 \mathrm{~J}, 400 \mathrm{fs}$ pulse at the fundamental wavelength of $1.053 \mu \mathrm{m}$ with an intensity contrast $5 \times 10^{5}: 1$. The $p$-polarized laser beam was focused on the surface of thin Mylar foil $6 \mu \mathrm{m}$ thick. The laser beam was incident at $45^{\circ}$ and focused down to a spot size of $\sim 12 \mu \mathrm{m}$ in diameter with an $f / 3(f=16.5 \mathrm{~cm})$ off-axis parabolic mirror. The maximum focused intensity was $6 \times 10^{18} \mathrm{~W} / \mathrm{cm}^{2}$. The maximum proton energy was measured with CR-39 plastic nuclear track detectors, placed parallel to the foil at a distance of $\sim 20 \mathrm{~cm}$ behind it. The detectors were covered with either steps of Mylar filters of thicknesses from 0 to $40 \mu \mathrm{m}$ or 25 to $525 \mu \mathrm{m}$ or steps of Mg filters with thicknesses from 250 to $1500 \mu \mathrm{m}$, depending on the expected maximum proton energy. The projectile ranges were calculated using the program SRIM2000. ${ }^{11}$ The maximum proton energy was found to vary linearly with the laser intensity (Fig. 1) and reaches 10 $\pm 1 \mathrm{MeV}$ at $6 \times 10^{18} \mathrm{~W} / \mathrm{cm}^{2}$. The proton energy given by the pondermotive potential of standing electromagnetic wave ${ }^{12}$

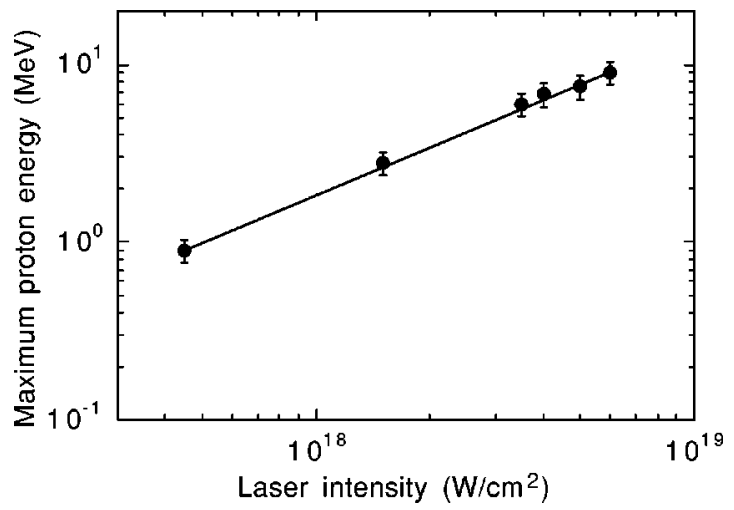

FIG. 1. Maximum proton energy in the forward direction as a function of laser intensity for $6 \mu \mathrm{m}$ Mylar foil. 


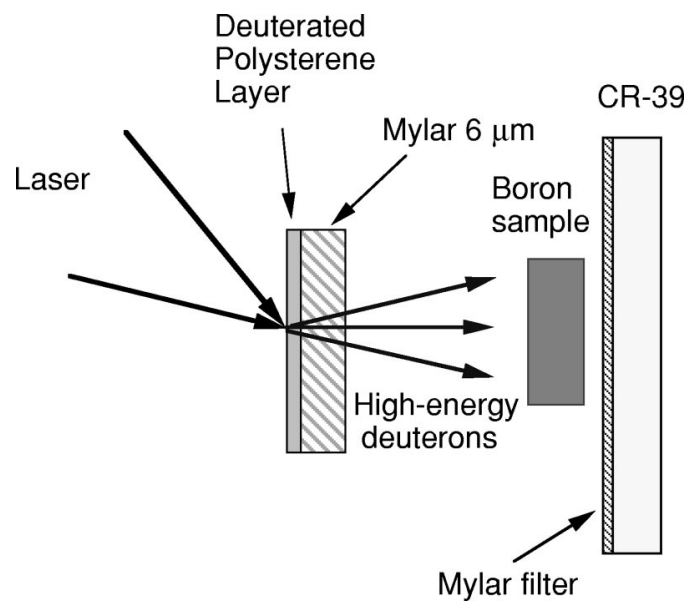

FIG. 2. Schematic diagram of the boron sample activation with high-energy deuterons.

is only $0.7 \mathrm{MeV}$, which is much less than the observed maximum proton energy. Estimation of the proton energy from the mechanism of "vacuum heating" 5 is closer to the observed value but also underestimates it by a few times. However, for the intensity contrast of $5 \times 10^{5}: 1$ it is questionable to apply the latter estimation because a preplasma very likely appears before the maximum laser energy reaches the target. Thus, one may attribute such a preplasma as a reason for an enhanced electron generation, in terms of energy and conversion efficiency, and, hence, for an enhanced electrostatic field which efficiently accelerates ions. Electrons accelerated in an underdense preplasma (electron density $n_{e}$ less than a critical) up to the energy $\epsilon_{\max }$ can penetrate inside a solid target to their Debye length, $\lambda_{\mathrm{De}} \propto \sqrt{\epsilon_{e} / n_{e}}$, and accelerate ions forward from the skin layer. Since the skin depth is shorter than $\lambda_{\mathrm{De}}$ a significant number of ions should have an energy equal to the electrostatic potential and the ion distribution function should demonstrate a plateau effect until energy cutoff $E_{\max }=Z \epsilon_{\max }$. Such an ion distribution function has already been observed in previous experiments. ${ }^{5}$

Deuterons should be accelerated less because they are two times heavier than protons, have less mobility and have higher energy losses. Since they follow behind the proton bunch, they experience a significantly smaller accelerating electric field. Consequently, they are expected to have a lower energy than protons. The high-intensity laser beam was focused on the Mylar target covered by a deuterated polystyrene layer. The resultant ion beam (protons and deuterons) was used to irradiate a ${ }^{10} \mathrm{~B}$ enriched sample (enrichment 90\%). A cylindrical shaped sample $10 \mathrm{~mm}$ in diameter and $5 \mathrm{~mm}$ thick was positioned on a CR-39 detector with a few steps of Mylar filters to monitor the energy of ion beam and its alignment. The deuterated polystyrene layer with a thickness $\gtrsim 1 \mu \mathrm{m}$ was deposited on the front side of the 6 $\mu \mathrm{m}$ Mylar film. The experimental setup is shown in Fig. 2. The concentration of boron isotope ${ }^{10} \mathrm{~B}$ in natural boron is $19.9 \%$. Therefore, in the enriched boron sample used the yield of isotope ${ }^{11} \mathrm{C}$ in the reaction ${ }^{10} \mathrm{~B}(d, n){ }^{11} \mathrm{C}$ must be enhanced approximately by a factor of 4.5 . The yield of ${ }^{11} \mathrm{C}$ was measured by counting the number of positrons emitted. The coincidence measurements require a low background count rate to receive a good signal to noise ratio. Two 3 in.

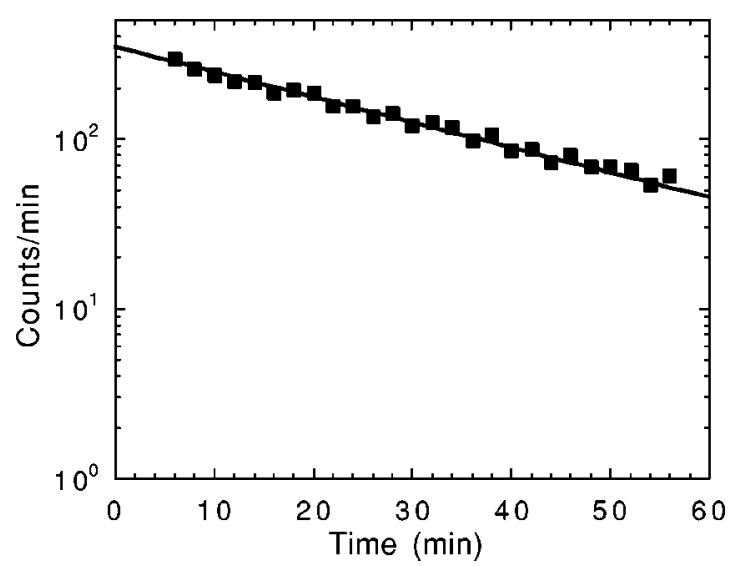

FIG. 3. Measured decay of radioactivity vs the time for the ${ }^{10} \mathrm{~B}(d, n){ }^{11} \mathrm{C}$ reaction (squares). The solid line corresponds to the theoretical result and demonstrates $\sim 20$ min half-life of ${ }^{11} \mathrm{C}$.

$\mathrm{Na}$ I detectors, placed opposite to each other, were used to detect the coincidence signal resulting from the annihilation of electrons and positrons and the production of two gammaquanta with energy of $0.511 \mathrm{MeV}$. The detection efficiency was measured to be in the range $0.05-0.1$ by using a ${ }^{22} \mathrm{Na}$ radioactive source with a precisely known activity of positron emission. The background noise was found to be 1-5 count/min and the radioactivity detection limit was $\sim 10 \mathrm{pCi}$.

Time dependence of a coincidence signal is shown in Fig. 3. We identified the source of this coincidence signal as the result of a positron decay of the carbon isotope ${ }^{11} \mathrm{C}$ with a half-life of $20.4 \mathrm{~min}$. We measured a maximum of $\sim 300$ counts/min at $330 \mathrm{~s}$ after the laser shot. The total yield of ${ }^{11} \mathrm{C}$ is estimated to be $\sim 10^{5}$ which corresponds to $\sim 2 \mathrm{nCi}$ of radioactivity immediately after the shot. The laser was able to deliver shots with a time interval of approximately 7 min. Therefore, accumulation of several shots will increase radioactivity by only a factor of three. If a similar laser had a repetition rate of $10 \mathrm{~Hz}$ and the irradiation time is equal to the half-life of ${ }^{11} \mathrm{C}$, a positron source with an activity of $\sim 20 \mu \mathrm{Ci}$ can be produced.

We have observed a very sharp power dependence of ${ }^{11} \mathrm{C}$ yield as a function of the laser intensity (Fig. 4) indicating that deuteron energies are near the threshold for the ${ }^{10} \mathrm{~B}(d, n){ }^{11} \mathrm{C}$ reaction. A significant fluctuation of ${ }^{11} \mathrm{C}$ yield at the maximum focused intensity can also lead to this con-

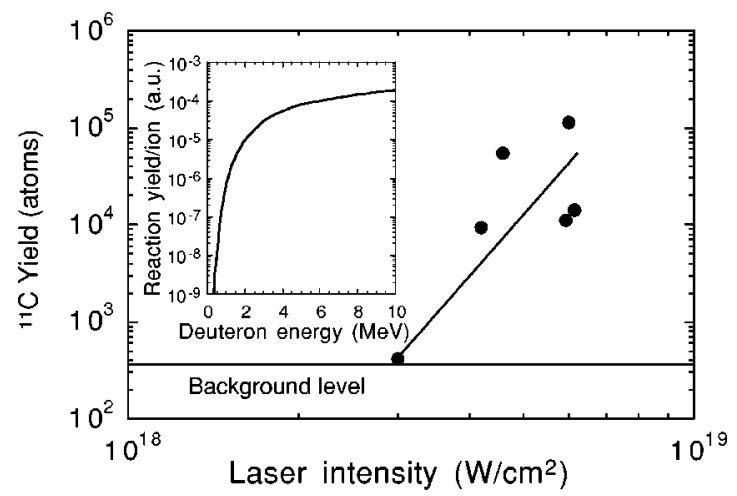

FIG. 4. Experimental yield of ${ }^{11} \mathrm{C}$ vs the laser intensity for the ${ }^{10} \mathrm{~B}(d, n){ }^{11} \mathrm{C}$ reaction. The inset shows calculated yield of ${ }^{11} \mathrm{C}$ per one deuteron as a function of energy cutoff $E_{\max }$ for a uniform distribution of deuterons in energy space. 
clusion. Reaction yield (per one accelerated ion) produced by an ion with charge $\mathrm{Ze}$ and reduced mass $M^{*}$ in a reaction with atoms having a density $n_{a}$ reads

$$
Y \equiv N / N_{i}=n_{a} \int_{0}^{\infty} d \epsilon \frac{d N_{i}}{d \epsilon} \int_{0}^{\epsilon} d \epsilon^{\prime} \sigma\left(\epsilon^{\prime}\right)\left|\frac{d \epsilon^{\prime}}{d r}\right|^{-1},
$$

where $N$ is the total number of reactions, $N_{i}$ is the number of accelerated ions, and bremsstrahlung losses are given by the Bete formula:

$$
\frac{d \epsilon}{d r} \simeq-\frac{M^{*}}{m \epsilon} 2 \pi e^{4} Z^{2} Z_{a} n_{a} \Lambda .
$$

Here, $m$ and $e$ are electron mass and charge, $\Lambda$ $=\ln \left(4 m \epsilon / M^{*} J\right)$, and $Z_{a}$ and $J$ are the atom charge and mean ionization potential of the medium atoms. We used the EXFOR data base ${ }^{13}$ for the ${ }^{10} \mathrm{~B}(d, n){ }^{11} \mathrm{C}$ reaction cross section and a uniform distribution of deuterons in energy space until energy cutoff $E_{\max }$ to determine ${ }^{11} \mathrm{C}$ yield from Eq. (1). The inset of Fig. 4 shows that the threshold for this reaction is $\sim 0.5 \mathrm{MeV}$. The comparison with the experimental data convinces one that the deuteron energy cutoff at the maximum laser intensity used is about $1-2 \mathrm{MeV}$.

To verify that deuteron acceleration is more efficient from the front side of the target we irradiated a $6 \mu \mathrm{m}$ thick Mylar target with a layer of a deuterated plastic on its back side and performed the same ${ }^{10} \mathrm{~B}$ activation experiment described above. In this case the activation signal was not above the background level. These results unequivocally indicate, that for the conditions of our experiment deuterons are accelerated from the front side of the foil or they acquire higher energy compared the rear side case.

High-energy protons are also expected in the experiment with the layered deuterated target. They may participate in the ${ }^{11} \mathrm{~B}(p, n){ }^{11} \mathrm{C}$ reaction. The cross section of this reaction ${ }^{13}$ has a higher threshold (above $3 \mathrm{MeV}$ ). Taking into account that the amount of ${ }^{11} \mathrm{~B}$ in boron sample was only $10 \%$, we expect ${ }^{11} \mathrm{C}$ yield for this reaction to be much less than that for deuterons. To verify that the $(p, n)$ reaction does not contribute sufficiently in ${ }^{11} \mathrm{C}$ yield, we focused the laser at its highest intensity on a bare Mylar film behind which was a boron sample. No activation signal was observed in this case.

In summary, we demonstrated the production of $\sim 10^{5}$ atoms of a positron active isotope ${ }^{11} \mathrm{C}$ by illumination of ${ }^{10} \mathrm{~B}$ sample with deuterons accelerated by high-intensity laser to energies of about $2 \mathrm{MeV}$. The activation results suggest that acceleration is more efficient from the front side of the target.

The work of some of the authors (A.M.), (K.F.), (G.M.) and the Laser Facility were supported by the U.S. NSF. The work of V.Yu.B. was supported by the Russian Foundation for Basic Research and that of D.U., by the Office of Energy Research, U.S. DOE. S.B. is thankful for the support of CUOS Fellowship Program and K.N. would like to thank S. Akita, S. Sasaki, and T. Suzuki for general support.

${ }^{1}$ E. O. Lawrence and N. E. Edlefsen, Science 72, 376 (1930).

${ }^{2}$ G. A. Mourou, C. P. J. Barty, and M. D. Perry, Phys. Today 51, 22 (1998).

${ }^{3}$ A. P. Fews, P. Norreys, F. N. Beg, A. R. Bell, A. E. Dangor, C. N. Danson, P. Lee, and S. J. Rose, Phys. Rev. Lett. 73, 1801 (1994).

${ }^{4}$ E. L. Clark, K. Krushelnick, J. R. Davies, M. Zepf, M. Tatarakis, F. N. Beg, A. Machacek, P. A. Norreys, M. I. K. Santala, I. Watts, and A. E. Dangor, Phys. Rev. Lett. 84, 670 (2000).

${ }^{5}$ A. Maksimchuk, S. Gu, K. Flippo, D. Umstadter, and V. Yu. Bychenkov, Phys. Rev. Lett. 84, 4108 (2000).

${ }^{6}$ S. P. Hatchett, C. G. Brown, T. E. Cowan, E. A. Henry, J. S. Johnson, M. H. Key, J. A. Koch, A. B. Langdon, B. F. Lasinski, R. W. Lee, A. J. Mackinnon, D. M. Pennington, M. D. Perry, T. W. Phillips, M. Roth, T. C. Sangster, M. S. Singh, R. A. Snavely, M. A. Stoyer, S. C. Wilks, and K. Yasuike, Phys. Plasmas 7, 2076 (2000).

${ }^{7}$ G. S. Sarkisov, V. Yu. Bychenkov, V. N. Novikov, V. T. Tikhonchuk, A. Maksimchuk, S.-Y. Chen, R. Wagner, G. Mourou, and D. Umstadter, Phys. Rev. E 59, 7042 (1999).

${ }^{8}$ T. Ditmire, J. W. G. Tisoh, E. Springate, M. B. Mason, N. Hay, R. A. Smith, J. Marangos, and M. H. R. Hutchinson, Nature (London) 386, 54 (1997).

${ }^{9}$ W. L. Kruer and K. Estabrook, Phys. Fluids 28, 430 (1985).

${ }^{10}$ F. Brunel, Phys. Rev. Lett. 59, 52 (1987).

${ }^{11}$ SRIM2000 by J. F. Ziegler, http://www.research.ibm.com/ionbeams/ home.htm

${ }^{12}$ S. C. Wilks, W. L. Kruer, M. Tabak, and A. B. Langdon, Phys. Rev. Lett. 69, 1383 (1992)

${ }^{13}$ Experimental Nuclear Reaction Data File, http://www.nndc.bnl.gov/nnde/ exfor 